\title{
Estudio sobre eficacia y carga de trabajo en hdf online post y hdf mixed
}

\author{
Mercedes Ureta Valer, Elena Herrera Martín
}

Hospital Universitario Infanta Leonor. Madrid

\section{Introducción:}

La óptima eficacia dialítica es uno de los mayores retos perseguidos por las unidades de nefrología. Múltiples estudios evidencian una importante disminución de la mortalidad. La aparición de nuevos monitores más sofisticados, nos permiten realizar técnicas más complejas adaptándose cada vez más a las necesidades de cada paciente. La HDF on line, es una técnica que ofrece una mayor depuración de pequeños y grandes solutos, al combinar en la transferencia los mecanismos de difusión y convección, utilizando tasas altas de ultrafiltración (612 litros/hora), que se compensan con la infusión on line de líquido de reposición; con mayor biocompatibilidad, ya que el líquido de infusión es ultrapuro, con todas las ventajas que ello conlleva. Sin embargo, nos encontramos con unos inconvenientes propios de la técnica como el aumento de la PTM con la posibilidad de coagulación del sistema. En nuestra unidad, contamos con un monitor de infusión Mixed controlada por feedback PTM-UF. Este feedback ajusta de forma automática la tasa y el lugar de infusión a la máxima fracción de filtración (FF) teniendo en cuenta las condiciones del flujo, presiones internas y permeabilidad hidráulica del dializador, y sus interacciones. El sistema regula el grado de infusión pre-post para mantener la PTM entre $250-340 \mathrm{mmHg}$.

\section{Objetivo:}

Comparar la eficacia dialítica a tiempo programado 0 efectivo en HDF post y Mixed, con idénticas membranas y esquema de tratamiento; así como valorar como repercuten la aparición de alarmas, tanto en la pérdida de eficacia dialítica como en el aumento de la carga de trabajo de enfermería.

\section{Método:}

Diseñamos un estudio prospectivo, randomizado y cruzado en el que se incluyeron 8 pacientes. Lo dividimos en 2 periodos de 6 sesiones en HDF Post y 6 en Mixed. En cada periodo, se realizaron 4 sesiones con un tiempo programado $\geq 240$ minutos y 2 sesiones en las que se alargó hasta un tiempo efectivo igual al previo programado. Las variables principales en el estudio han sido el tiempo, la técnica y los parámetros dialíticos. También contabilizamos el número de alarmas intradiálisis.

\section{Resultados:}

Tras analizar las sesiones evidenciamos una disminución significativa del tiempo efectivo de diálisis respecto al tiempo programado. Comparando ambas técnicas, la HDF Post pierde una media de 4,6 minutos de eficacia mientras que en HDF Mixed, se pierde una media de 9,2 minutos; disminuyendo significativamente el $\mathrm{Kt}$ $(p=0,003)$ en HDF Mixed. Tras analizar las sesiones en tiempo efectivo no se evidencian diferencias significativas entre la eficacia dialítica en HDF Mixed o Post $(p=0,426)$. Tras analizar el tiempo efectivo y $n^{\circ}$ de alarmas no se evidencia ninguna correlación $(p=0,75)$.

\section{Conclusiones:}

Valorando los resultados obtenidos, concluimos que el factor tiempo es determinante en la eficacia dialítica. A mayor tiempo programado mejores parámetros dialíticos sin influir la técnica HDF elegida. Aunque no hay correlación entre tiempo efectivo y $n^{\circ}$ de alarmas, sí hemos podido objetivar que en 
casos aislados de riesgo de hemoconcentración, el $\mathrm{n}^{\circ}$ de alarmas relacionadas son inferiores en Mixed que en Post, disminuyendo la carga de trabajo enfermera.

\section{Referencias Bibliográficas}

1. García H, Hernández-Jaras J, Maduell F, Yago M, Calvo C, Navarro V. et.al Eficacia de la hemodiafiltración en línea (HDF) comparada con la hemodiálisis de alto flujo (HD). NEFROLOGÍA. 1998; 18(6): 476-482.
2. Terry Osset $\mathrm{C}$, Párraga Díaz $\mathrm{M}$, Cárceles Legáz $\mathrm{E}_{\text {, }}$ Belchí Rosique EF, Astorga Pérez F, Chica Arellano J. Estudio comparativo de la eficacia de dos técnicas de HDF on line. Rev Soc Esp Enferm Nefrol 2006; 9 (4): 249/253.

3. Fernández Martínez AV, Piñero Martínez J, Arregui Arias Y, Pérez García R, Casanova Arroyo M, Rodríguez Martínez F. et. al. Dializar en tiempos de crisis: comparación de la eficacia dialíticamente una pauta estándar frente a otra optimizando flujo sanguíneo y tiempo efectivo en el paciente en hemodiafialtración on-line. Enferm Nefrol 2012; 15 Suppl (1): 17/83. 